\title{
El Aristoteles Sinicus: Examen del comentario aristotélico en chino de la primera Edad Moderna desde una perspectiva comparativa
}

\section{Isabelle Duceux*}

BOSTON COLLEGE

Este artículo se propone estudiar la adaptación del pensamiento aristotélico al chino por los misioneros jesuitas al inicio del siglo XvII. Para pensar la especificidad de este encuentro cultural e intelectual, se comparan los comentarios aristotélicos redactados en chino con los comentarios de los jesuitas tanto en Europa como en América. Esto lleva a entender la adaptación como una suerte de acomodación y a subrayar en qué medida los rasgos culturales influyen sobre las modalidades del encuentro.

(Jesuitas, filosofía aristotélica, comentarios, China, encuentros culturales)

\section{INTRODUCCIÓN}

- a historia de los comentarios aristotélicos es larga y rica. Desde la obra de Aristóteles del siglo Iv a.C. hasta el Lingyan lishao de Francesco Sambiasi (1582-1649), el comentario jesuítico al De anima publicado en 1624 , se pueden contar veinte siglos de comentarios e interpretaciones de la obra de Aristóteles, los cuales se redactaron en diversos idiomas: el griego, el árabe, el latín y por último el chino. El tipo de comentario que nos interesa aquí se remonta a la Edad Media y nace de la lectio, la cual consistía en la lectura y explicación en voz alta de un texto. ${ }^{1} \mathrm{La}$ Biblia, las Sentencias de Pedro Lombardo y el corpus aristotelicum contaban dentro de

*dumeiyi@hotmail.fr

${ }^{1}$ Alain de Libera, "De la lecture à la paraphrase; remarques sur la citation au Moyen Âge”, Langage, núm. 73, 1984, 17-29. 
las obras más comentadas. ${ }^{2}$ En sí, la razón de los comentarios era exponer y explicitar el contenido filosófico o teológico de una obra. Asimismo, se pueden definir como obras de interpretación y de crítica. ${ }^{3}$ Este punto es de suma relevancia porque pone en evidencia la distancia que existe entre el texto original y el comentario. Esta distancia resultó en primera instancia de la modificación que el mundo cristiano impuso a la lectura de Aristóteles, la cual generó la necesidad misma del comentario. Claro es que "el aristotelismo medieval no podía ser el de Aristóteles sencillamente porque vivía en un mundo diferente, en un mundo en el cual se sabía que no había y no podía haber más que un solo Dios". ${ }^{4}$ Otro factor que explica la distancia entre la obra comentada y el comentario es la tradición filosófica en la cual se concibe el comentario mismo, plasmando las diversas influencias e intereses propios de un momento intelectual e histórico específico. Asimismo, los comentarios aristotélicos del Renacimiento hacen referencia no sólo a santo Tomás (1225-1274) sino también a los autores patrísticos, a Platón, a los neoplatónicos desde Plotino (204/5-270) hasta el mítico Hermes Trismegisto, y finalmente a la cultura humanista propia de los siglos XV y XVI.

Los jesuitas fueron prolijos redactores de comentarios a la obra de Aristóteles. Esas peculiaridades de los comentarios aristotélicos que acabamos de mencionar se encuentran, tanto en los comentarios del viejo continente, como en los de América y Asia. Un análisis detallado de las obras muestra el mismo uso de las referencias con algunas diferencias según los autores. Lo anterior no quiere decir que los tratados redactados en chino fueran exactamente similares a los demás tratados publicados en otros continentes. En este artículo, nos proponemos analizar las características propias de los comentarios redactados en chino por los misioneros jesuitas desde una perspectiva comparativa. Por tanto se examinaran tres comentarios al De anima de Aristóteles, los Commentarii Collegii Conimbricensis Societatis Iesu, In tres libros de Anima Aristotelis Stagiritae de Manuel de

${ }^{2}$ Etienne Gilson, La philosophie au Moyen Age, p. 38.

${ }^{3}$ Etienne Gilson, La philosophie au Moyen Age, p. 15.

${ }^{4}$ Alexandre Koyré, "Aristotélisme et platonisme dans la philosophie du Moyen Âge", Études d'histoire de la pensée scientifique, París, Gallimard, Tell, 1973, 39. 
Góis (Coimbra, António de Mariz, 1598); los Commentarii in libros Aristotelis Stagiritae Philosophorum Principis de Anima de Antonio Rubio (Alcalá 1611); y el Lingyan lishao (Humilde discusión sobre cuestiones del alma) de Francesco Sambiasi (¿Shanghai, Jiading o Hangzhou? 1624).

\section{Los Commentari Collegil Conimbricensis Societatis IEsu}

Los Commentarii Collegii Conimbricensis Societatis Iesu 5 son una serie de 8 tratados redactados entre 1592 y 1606 para facilitar la enseñanza del corpus aristotelicum en el Colegio de Arte de Coimbra y la Universidad de Évora en Portugal. Son varias las razones que motivaron la redacción de los comentarios jesuitas de Coimbra. ${ }^{6} \mathrm{La}$ idea que estos cursos pudieran fungir como apoyo para los profesores y estudiantes en la clase fue de Jerónimo Nadal (1507-1580) y surgió en 1561. Al tener unos libros de texto, los estudiantes serían liberados de la toma de nota y más dedicados a la asimilación de los contenidos. Otra razón era la de crear una unidad doctrinal. Los

${ }^{5}$ Commentarii Collegii Conimbricensis Societatis Jesu in octo libros Physicorum Aristotelis Stagyritae (Coimbra, A. Mariz, 1592; Lyon, 1594); Commentarii Collegii Conimbricensis Societatis Iesu in quattuor libros De Coelo Aristotelis Stagiritae (Lisboa, S. Lopes, 1593); Commentarii Collegii Conimbricensis S. I. in libros Metereororum Aristotelis Stagiritae (Lisboa, S. Lopes, 1593); Commentarii Collegii Conimbricensis S. I. in libros Aristotelis qui Parva Naturalia appellantur (Lisboa, S. Lopes, 1593); In libros Ethicorum Aristotelis ad Nicomachum aliquot Conimbricensis Cursus disputationes, in quibus praecipua quaedam Ethicae disciplinae capita continentur (Lisboa, S. Lopes, 1593); Commentarii Collegii Conimbricensis S. I. in duos libros de generatione et corruptione Aristotelis Stagiritae (Coimbra, A. Mariz, 1597); Commentarii Collegii Conimbricensis S. I. in tres libros De anima Aristotelis Stagiritae (Coimbra, A. Mariz, 1598); Commentarii Collegii Conimbricensis S. I. in universam Dialecticam Aristotelis (Coimbra, D. G. Loureiro, 1606). Para una presentación detallada de las múltiples ediciones de los cursos, véase Antonio Alberto de Andrade, Curso Conimbricense. P Manuel de Góis, Moral a Nicómaco, de Aristóteles, Introducción, Lisboa, Instituto de Alta Cultura, 1957, XVI-XIX.

${ }^{6}$ Sobre las designaciones comunes de "Curso Conimbricense" o "Conimbricenses", Mario Santiago de Carvalho menciona que no son adecuadas porque otras ordenes religiosas tenían en esta misma ciudad su producción de comentarios, los cuales también se podrían denominar "Conimbricenses". Véase Mario Santiago de Carvalho, "Tentâmen de sondagem sobre a presença dos platonismos no volume do De Anima do primeiro Curso Jesuíta Conimbricense" en José Antônio de Camargo Rodrigues de Souza, Idade Média: tempo do mundo, tempo do homens, tempo de Deus, Porto Alegre, Est Ediçóes, 2006, 391. 
pensamientos de Aristóteles y santo Tomás formaban la base de la enseńanza de la Compañía de Jesús. ${ }^{7}$ El aristotelismo y su interpretación medieval por Tomás de Aquino fueron las doctrinas escogidas por los fundadores de la Compañía con el fin de luchar contra las ideas reformadas. El curso de tres años empezaba con la lógica después de la cual se enseñaba la filosofía natural y la metafísica. ${ }^{8} \mathrm{La}$ teología coronaba esta formación. Estos comentarios también permitirían excluir los puntos incompatibles con la doctrina de la Iglesia. ${ }^{9}$ Finalmente, los jesuitas se enfrentaban al problema de la multiplicación de las interpretaciones de Aristóteles que se dieron con la revolución lingüística propia de la época. En efecto, el Renacimiento favoreció una lectura renovada de los textos clásicos basada en traducciones directas del griego y un estudio filológico detallado de estos textos. Por lo mismo, Pedro da Fonseca (1528-1599) ideó el curso completo de la obra aristotélica, los Cursus Collegii Conimbricensis Societatis Iesu, para dar una lectura ortodoxa y fomentar una unidad intelectual en el seno de la Compañía.

Los cursos presentaban el texto aristotélico en latín -sólo ciertas ediciones foráneas ofrecían el texto griego original $-{ }^{10} \mathrm{y}$ un comentario (explanatio) cuyo fin era explicitar el texto aristotélico mediante la paráfrasis. ${ }^{11}$ Es notable que la paráfrasis a menudo incluyera largas citas de autores antiguos y medievales. Luego se presentaban las quaestiones. La quaestio consistía en la discusión de un problema y la refutación de posiciones divergentes. Los argumentos se fundamen-

${ }^{7}$ Véase, Charles Lohr, "Les jésuites et l'aristotélisme du xvıe siècle" en Luce Giard, Les jésuites à la Renaissance: système éducatif et production du savoir, París, PUF, 1995, 80; Paul F. Grendler, "The Universities of the Renaissance and Reformation”, Renaissance Quarterly, vol. 57, núm. 1, 2004, 25-26.

${ }^{8}$ Cabe notar aquí que a partir de 1552 , los estatutos de las universidades de Coimbra y Évora, aprobados por el rey de Portugal, preconizaban un curso de filosofía de 4 años y no 3. Véase, Antonio M. Martins, "The Conimbricenses. Introductory note to the Commentarii Collegii Conimbricensis Societatis Iesu”, Biblioteca Saavedra Fajardo de Pensamiento Político Hispánico, p. 2.

${ }^{9}$ Martins, “The Conimbricenses. Introductory note...” p. 3.

${ }^{10} \mathrm{Tal}$ es el caso de la edición que consultamos, Colonia, Lazari Zetzneri, 1617.

${ }^{11}$ Para este párrafo me apoyo en Martins, "The Conimbricenses. Introductory note..." p. 12. 
taban en la autoridad, la razón, la fe y la experiencia. ${ }^{12}$ En los cursos jesuíticos de Coimbra, las quaestiones ya no siguen la estructura de la obra aristotélica. Es en ellas que debe encontrarse la sustancia del pensamiento jesuítico. Alison Simmons asevera al respecto:

Las quaestiones son impresionantes no solamente por su contenido original sino también por el modo de presentación o el estilo del argumento que se podría clasificar hoy en día como una reconstrucción racional; es decir, reconstruyen efectivamente el pensamiento de Aristóteles desde los primeros principios o por lo menos los principios relativamente fundamentales de su propio sistema filosófico. ${ }^{13}$

Finalmente, los argumentos empleados eran a veces no aristotélicos o hasta contrarios al pensamiento de Aristóteles. Por otra parte, agregaban discusiones relativas a otros campos que la filosofía tales como las matemáticas o la medicina. En este sentido, el De anima de Coimbra es un buen ejemplo, ya que el primer capítulo expone las doctrinas del alma de los fisiólogos, los médicos, dialécticos y matemáticos. ${ }^{14}$ Por lo anterior, Antonio Martins concluye que "es un anacronismo totalmente injustificado el describir esos textos como 'medievales' lo que sigue ocurriendo hoy en día". ${ }^{15}$ Lo mismo se subraya hablando del tomismo de la Contrarreforma en general ${ }^{16}$ y específicamente del De anima de Coimbra: ${ }^{17}$

${ }^{12}$ Katharine Park, "The organic Soul” en Charles B. Schmitt, Quentin Skinner y Eckart Kessler, The Cambridge History of Renaissance Philosophy, Cambridge, Cambridge University Press, 1998, 473.

${ }^{13}$ Alison Simmons, "Jesuit Aristotelian Education: The De anima Commentaries" en John W. O’Malley, Gauvin Alexander Bailey, Steven J. Harris y T. Frank Kennedy, eds., The Jesuits: Cultures, Sciences, and the Arts, 1540-1773, Toronto, Bufalo, Londres, University of Toronto Press, 1999, 526.

${ }^{14}$ Commentarii Colegii Conimbricensis, Societatis Iesu: In Tres Libros de Anima, Aristotelis Stagiritae, libro I, cap. 1: "Scientiae de anima dignitas: methodus inueniendi animae definitionem: ac modus, quem in definiendo seruent Physiologus, Medicus, Dialecticus, et Matematicus".

${ }^{15}$ Martins, "The Conimbricenses. Introductory note..." p. 12.

${ }^{16}$ Katharine Park y Eckard Kessler, "The Concept of Psychology" en The Cambridge History of Renaissance Philosophy, chap. 13.

${ }^{17}$ Mario Santiago de Carvalho, Psicologia e ética no curso jesuita conimbricense, Lisboa, Ediçóes Colibri, Forum de Ideias, 2010, 31. 
En lo que toca a la psicología, [los comentarios] compartían completamente una situación renacentista singular. Esto significa que la revitalización de la filosofía del siglo XIII, hecha a la luz de la sofisticación filológica de los Humanistas, también ante las nuevas fuentes griegas, con el apoyo manifiesto de los poderes de la imprenta ('omnia omnibus' es el significativo lema del editor portugués João de Barreira), no representa una absoluta ruptura con la filosofía de los siglos XIV y xV, y confiere a aquellos Comentarios la particularidad de contribuir a una relectura de la doctrina de Aristóteles promovida en la variedad de una tradición comentarista más amplia y que se acababa de redescubrir.

Esta cultura humanista permea el De anima de Manuel de Góis (1547-1599). Lo vemos tanto en el énfasis que pone en el carácter fisiológico del alma como en sus referencias. Asimismo, la insistencia de los comentaristas sobre las cuestiones relativas al alma orgánica, en particular, la naturaleza de la percepción sensible, el proceso de generación y la relación entre el cuerpo y el alma, indican que eran temas ampliamente debatidos por los pensadores del siglo Xvi. ${ }^{18}$ En este sentido, el comentario al De anima de Coimbra dedica un número más grande de cuestiones a la teoría del conocimiento sensible (35) que al intelecto (14). ${ }^{19}$ Es más, de acuerdo con el comentario se puede afirmar que la indagación de la naturaleza o esencia del alma compete a la fisiología por tres razones:

a) Porque, al llevar en serio la definición del alma como acto primero de un cuerpo orgánico, es necesario incluir el conocimiento de la materia en el estudio de la esencia del alma; b) porque la propia respuesta a la pregunta “¿qué es el hombre?” en cuanto éste es participante de los seres animados depende obviamente de la física; c) porque la caracterización del ser humano como constituido de un cuerpo y participando de un espíritu racional es asunto exclusivo de la física. ${ }^{20}$

${ }^{18}$ Park, “The organic Soul”, pp. 473-474.

${ }^{19}$ Carvalho, Psicologia e ética no curso jesuita conimbricense, p. 33.

${ }^{20}$ Carvalho, Psicologia e ética no curso jesuita conimbricense, p. 40. 
Lo anterior lleva a examinar las referencias que fundamentan los argumentos y contraargumentos del comentario conimbricense al De anima. Estas pertenecen a una amplia gama de tradiciones filosóficas, no siempre fieles al pensamiento del Estagirita. Vemos por ejemplo que todos los "platonismos"21 se encuentran mencionados en el curso jesuítico de Coimbra, es decir Platón mismo (Timeo, Fedro, Leyes, etcétera), los neoplatónicos empezando por Plotino y Porfirio (232-304), la patrología griega y latina (san Agustín (354-430), Pseudo-Dionisio (entre los siglos v y vi d. C.), Orígenes (185-254), etcétera), los platónicos medievales (san Bernardo (1090-1153), Boecio (480-525), etcétera) y finalmente los platónicos del Renacimiento (Hermes Trismegisto, Ficino (14331499), Pomponazzi (1462-1525), etcétera). ${ }^{22}$ Por otra parte, la referencia a Averroes está presente en todos los comentarios jesuíticos al De anima. Averroes examina ¿cómo un intelecto material engendrado y entonces corruptible como lo es el intelecto humano podrá tener un conocimiento de los inteligibles que son eternos? $\mathrm{Su}$ solución consistió en hacer del intelecto agente un intelecto separado del alma humana y concluyó que el intelecto agente no pertenece a la definición aristotélica del alma como forma del cuerpo. Asimismo, Averroes definió que lo que es inmortal en el hombre no es más que lo que pertenece al intelecto agente, es decir nada proveniente del alma humana en $s^{i}{ }^{23}$ Mediante estas referencias, advertimos que el De anima de Coimbra es un comentario renacentista en la medida en que integra no solamente la cultura humanista sino también las preocupaciones propias del siglo xvi. No se puede hablar entonces de un comentario lineal de la obra de Aristóteles o de santo Tomás.

${ }^{21}$ La palabra "platonismos" en plural propuesta por Mario Santiago de Carvalho hace eco a Ch. Schmitt quien primero habló de "aristotelismos" para describir la variedad de interpretación del pensamiento de Aristóteles durante el Renacimiento.

${ }^{22}$ Carvalho, "Tentâmen de sondagem..." pp. 394-395.

${ }^{23}$ Etienne Gilson, La filosofía en la Edad Media, Madrid, Gredos, 1985, 341-342. 


\section{Antonio Rubio y la filosofía jesuítica en México}

Con Antonio Rubio (1548-1615), abordamos un segundo comentario jesuítico al De anima, el Commentarii in libros Aristotelis Stagiritae Philosophorum Principis de Anima publicado por primera vez en 1611 en la cuidad de Alcalá. ${ }^{24}$ El padre Antonio Rubio era oriundo de La Roda (Albacete, España). El hecho de que se formó en la universidad de Alcalá es notable porqué Alcalá era uno de los centros universitarios más importantes de España en cuanto a la restauración escolástica. Santo Tomás era el pensador de referencia de esta restauración. No es de sorprender entonces que Rubio fuera un discípulo intelectual del Aquinate, lo que expresa en el epílogo de su comentario del De anima: "Y baste lo dicho acerca de estos comentarios a Aristóteles hechos para alabanzas y gloria de la Santísima Trinidad, de la Santísima Madre de Dios y del Angélico Doctor cuya doctrina he seguido y defendido como humilde discípulo". ${ }^{25}$ La Compañía de Jesús lo mandó a México donde llegó en 1576. En México, enseñó la filosofía por 4 años y la teología por 12. Antonio Rubio tenía expectativas altas y quería escribir un comentario exhaustivo de la obra de Santo Tomas.

El sobredicho P. Antonio Rubio ha leído muchos ańos philosophia y teología con gran aceptación y satisfacción: desea hazer un Comentario sobre las partes de Sancto Thomás, que no sea cuestionario, sino verdadero comento que declare el sentido de Sancto Thomás, y en cual se defiendan y apoyen todas sus opiniones, sacándolas de sus primeras rayces y fundamentos, y se concuerden diversos lugares suyos; [...] V. R. se contente dar esta licencia, y remitirla, que acá la vean los Padres Plaza, Pedro Sánchez, Pedro de Hortigosa, Loysa, o de los que destos paresciere, y aprobado, se imprima. ${ }^{26}$

${ }^{24} \mathrm{El}$ texto al cual referimos es la edición de 1613 publicada por el editor Pillehotte en Lyon, Francia.

${ }^{25}$ Citado por Camilo Falcon Gyves, El P. Antonio Rubio, S. J. (1548-1615): sus comentarios a los libros "De Anima" de Aristóteles, México, Bajo el signo de "Ábside”, 1945, 22.

${ }^{26}$ Citado por Juan Carlos Torchia Estrada, "El Padre Antonio Rubio y la enseńanza filosófica de los jesuitas en la Nueva España”, CUYO, Anuario de Filosofía Argentina y Americana, núm. 13, 1996, 22-23. 
Dentro de las razones que lo motivaron a redactar este curso, volvemos a encontrar el deseo de liberar a los estudiantes de la "tediosa" toma de nota y facilitar por lo tanto un verdadero intercambio intelectual durante la clase. ${ }^{27} \mathrm{Su}$ obra mayor es un curso de filosofía en cinco volúmenes dedicados al comentario de los textos aristotélicos, el Organon, la Física, el De anima, el De generatione et coruptione y el De caelo et mundo. ${ }^{28}$ Rubio también tenía considerada la edición de un comentario a la Metafísica pero no logró publicarla. Es notable el hecho de que no se percibiera en la obra de Rubio y en general de los pensadores novohispanos su ubicación en el mundo americano. En este sentido Walter Redmond asevera que "estos escritores se involucraban en asuntos prácticos de América, pero estos asuntos no interferían en su filosofía académica. Lo anterior es cierto también de Antonio Rubio, aunque en este caso un conflicto se percibe entre el escribir y enseñar 'aquî' y 'allá', en la Nueva y la Vieja Espańa”. ${ }^{29}$

El De anima del padre Rubio se divide en tres partes al igual que la obra del Estagirita y que el De anima de Coimbra. Ahora bien, una diferencia considerable con los comentarios tradicionales se observa en la presentación de la obra de Rubio: el texto comentado, es decir, la obra de Aristóteles, ya no se reproduce salvo en la Lógica donde su reproducción es sin embargo muy breve. ${ }^{30}$ En cuanto a la estructura del comentario, encontramos elementos similares al $D e$ anima de Manuel de Góis: el comentario del texto aristotélico se

${ }^{27}$ Walter Redmond, "Self-Awareness in Colonial Latin American Philosophy" Jahrbuch für Geschichte Lateinamerikas 41, Böhlau Verlag, Köln, Weimar, Wien, 2004, 370. La referencia es Rubio, Lógica, "Auctoris ad lectorem praefatio".

${ }^{28}$ Para los párrafos siguientes, me refiero principalmente a la obra de Falcon Gyves, El P. Antonio Rubio...

${ }^{29}$ Redmond, "Self-Awareness in Colonial Latin American Philosophy...” p. 357.

${ }^{30}$ Sobre la presentación física de los comentarios, véase, Louis Holtz, "Le rôle des commentaires d'auteurs classiques dans l'émergence d'une mise en page associant texte et commentaire (Moyen Âge occidental)" en Marie-Odile Goulet-Cazé, Tiziano Dorandi, coords., Le Commentaire entre tradition et innovation, Actes du Colloque International de l'Institut des Traditions Textuelles, Paris et Villejuif, 22-25 octubre 1999, París, Librairie Philosophique J. Vrin, 2000, 101-116; Torchia Estrada, "El Padre Antonio Rubio y la enseñanza filosófica..." p. 24. 
reduce a una paráfrasis de 3 a 4 páginas por capítulo mientras que el desarrollo de las quaestiones es extenso. Escribe Rubio:

Desplegamos el texto de Aristóteles por capítulo, o al menos el comienzo de cada uno, en la traducción latina de Boecio Severino. [...] Agregaremos nuestra explicación para aclarar el texto y entonces algunas notas si en alguna parte lo pide la importancia o dificultad de la materia, y finalmente llamaremos en controversia toda la doctrina misma de Aristóteles, para que su verdad se haga más lúcida y evidente. ${ }^{31}$

También tiene un número amplio de referencias. Cita tanto a los teólogos dominicos que estudió durante su formación en Alcalá como a los teólogos jesuitas. ${ }^{32}$ Las referencias a los Padres de la Iglesia son previsibles ya que la enseñanza que recibían los jóvenes jesuitas se basaba primeramente en la escolástica tomista la cual se completaba con una teología positiva que consistía en las obras de los Padres de la Iglesia. ${ }^{33}$ Menciona a Platón, a Temistio (317-390), exégeta del platonismo y al neoplatónico Simplicio (490- c. 560) cuyo De anima provocó mucha discusión y controversia durante el Renacimiento. ${ }^{34}$ También es oportuno subrayar el hecho de que el estudio del alma era previo a los estudios superiores de medicina. Es predecible entonces que se encuentren referencias a Galeno (130200) en los comentarios del De anima. Alberto Magno (1193/12061280) y san Buenaventura (1218-1274) figuran dentro de los pensadores medievales que Rubio nombra. Pomponazzi y Ficino son algunos de los pensadores renacentistas igualmente representados en la obra de Rubio. Finalmente disputa las teorías de los pen-

${ }^{31}$ Torchia Estrada, “El Padre Antonio Rubio y la enseñanza filosófica...” p. 26.

32 Falcon Gyves, El P. Antonio Rubio..., p. 23. Los teólogos dominicos que nombra son Francisco de Vitoria (1492-1546), Domingo Soto (1494-1560) y Domingo Báñez (1528-1604). Mientras que los teólogos jesuitas son Gabriel Vázquez (1549 o 1551 1604), Pedro da Fonseca (1528-1599), Francisco Suárez (1548-1617) Francisco de Toledo (1532-1596) sin olvidar los "Conimbricenses".

${ }^{33}$ Menciona a san Agustín, Dionisio (siglo III), Gregorio de Nisa (335-395) y Tertuliano (c. 160-c. 220).

${ }^{34}$ Charles Schmitt, Aristotle and Renaissance, Cambridge, Harvard University Press, $1983,92$. 
sadores árabes, Avicena (980-1037) y Averroes (1126-1198) en particular. Lo anterior invita a subrayar el hecho de que la psicología renacentista es heredera tanto del aristotelismo "medieval" como del neoplatonismo griego, la medicina de Galeno y los comentarios árabes.

Dentro de las cuestiones más estudiadas por Antonio Rubio se encuentra la cuestión del alma orgánica y la cuestión correlativa del conocimiento sensible. En la edición de 1613 (Lyon) Rubio dedica las páginas 278-309 al alma vegetativa, 309-651 al alma sensitiva y 652-771 al alma racional. Esto confirma el interés de los pensadores renacentistas por el alma orgánica y los procesos cognoscitivos que ya hemos recalcado al abordar el De anima de Coimbra. Finalmente, al igual que la obra de Manuel de Góis, el De anima de Rubio no abunda en el tema de la voluntad. Asimismo, el "Tratado de la voluntad" cuenta apenas con 2 cuestiones en 10 páginas (772-782) en una obra de 783 páginas (sin contar el "Tratado del alma separada" (783-821)). En realidad, Rubio examina el alma orgánica dentro del ámbito de la filosofía natural, es decir, la física, mientras que subsume el alma racional separada del cuerpo al Tratado del alma separada.

\section{Características del Comentario al DE anima Redactado EN CHINO}

Estas mismas referencias que hemos encontrado tanto en el De anima de Manuel de Góis como en el de Antonio Rubio se encuentran parcialmente en la obra de Francesco Sambiasi. El número limitado de referencias se debe al hecho de que los misioneros jesuitas en China rara vez mencionaban sus fuentes occidentales ya que eran desconocidas por sus lectores chinos. De ahí se puede concluir que cuando se citaban algunos autores era probablemente con la meta de enseñar a los catecúmenos. Aparte de Aristóteles y Tomás de Aquino, el autor cita explícitamente a Bernardo de Claraval y a Plutarco e implícitamente a Averroes y a la teoría de la circulación de la sangre que se estaba gestando en estos mismos años, antes de que William Harvey publicara su obra, De motu cordis et sanguinis en 1628. Notable es el hecho de que Sambiasi explicitara la noción de 
gracia refiriéndose a san Agustín más que a santo Tomás cuando todo el resto del comentario se apoya en el Doctor Angélico. ${ }^{35}$ Es cierto que la teoría de la gracia de santo Tomás es muy similar a la de san Agustín. Cabe subrayar, sin embargo, que el problema de la gracia fue central en la teología del Renacimiento precisamente porque era uno de los puntos sobre el que más contendían los católicos y los reformados. Ahora bien, Lutero y los reformadores fundamentaron su pensamiento en san Agustín, disociando sin embargo la fe y la razón que san Agustín vinculaba en el crede ut intelligas. Es entonces muy probable que Sambiasi se refiera explícitamente a san Agustín para insistir en el verdadero sentido de la gracia tal como el obispo de Hipona lo había formulado.

La primera discrepancia entre los textos jesuíticos publicados en Europa y América con los publicados en China se refiere a la presentación gráfica de las obras. Ya no se puede realmente hablar de comentario porque el texto de referencia ha desaparecido. Sin embargo, Sambiasi queda fiel a ciertos aspectos del comentario tradicional. Asimismo, centra sus análisis sobre las quaestiones y las termina con una refutación de las opiniones erróneas más debatidas como, por ejemplo, la teoría que identificaba la sangre y el alma o la teoría de los espíritus según la cual el alma era parte de la sangre. ${ }^{36}$ Una segunda diferencia reside en el hecho de que Sambiasi sigue con bastante cercanía la Suma teológica del Aquinate más que el De anima de Aristóteles. ${ }^{37}$ Asimismo, el Lingyan lishao se divide en 2 partes (anterior y posterior según una división china) examinando en primer lugar el alma en cuanto objeto de la epistemología y luego de la teología. De igual manera, cada parte se divide en dos subcapítulos. Escribe Sambiasi: "El presente tratado habla del ánima en cuatro capítulos. En primer lugar, se trata de la sustancia del ánima, en segundo lugar, se trata de las facultades del ánima, luego, de la dignidad del ánima y por último del objeto de las afecciones del so-

${ }^{35}$ Para este párrafo, véase Isabelle Duceux, La introducción del aristotelismo en China a través del De anima, siglos XVI-XVII, México, El Colegio de México, 2009, 63-66.

${ }^{36}$ Lingyan lishao, p. 22.

${ }^{37}$ Para un análisis del Lingyan lishao, véase Duceux, La introducción del aristotelismo en China... 
berano bien". ${ }^{38}$ Vemos entonces que el Lingyan lishao pone mucho énfasis en el problema teológico contrariamente a Manuel de Góis y Antonio Rubio quienes reservan este tópico para el Tratado del alma separada. En el Lingyan lishao, la división del alma entre un concepto epistemológico y otro teológico se plasma en las palabras utilizadas porque Sambiasi distingue las almas vegetativa, sensitiva e intelectiva (shenghun, juehun y linghun) del concepto de alma con una connotación teológica que llama en chino yanima. ¿Por qué esta distinción? Tal vez se debe al problema de la traducción. El vocablo que Sambiasi utilizó para traducir el término de alma ( hun) en el sentido gnoseológico es una palabra que pertenece al ámbito de la literatura china porque el alma no es una noción filosófica en China. Es probable que Sambiasi quiso evitar cualquier tipo de mala interpretación en cuanto a la noción teológica del alma por lo cual forjó el término transliterado de yanima (ánima). Esta noción teológica apunta al alma en cuanto se asemeja a Dios, en particular, por su voluntad y su alma racional e inmortal.

Otra diferencia significativa tiene que ver con el idioma de redacción. Las constituciones de la Compañía de Jesús incitaban a aprender la lengua del país de la misión. Es así como los jesuitas fueron pioneros en el campo de la lingüística redactando los primeros diccionarios y gramáticas de idiomas indígenas para el uso misional. La gramática del náhuatl de Antonio del Rincón (15661601) y el diccionario y la gramática del idioma otomí de Antonio de Ágreda (1714-1785) respectivamente publicados en México en 1595 y 1769 son unos ejemplos del trabajo lingüístico de los jesuitas. También se realizaron varios diccionarios del chino. Asimismo, Michele Ruggieri (1543-1607) y Matteo Ricci (1552-1610) publicaron un diccionario portugués-chino al final del siglo Xvı y Nicolas Trigault (1577-1628) redactó un Diccionario de la pronunciación china y europea (Hangzhou, 1626). De igual manera, esta política lingüística favoreció la redacción de obras de teología en lenguas

${ }^{38}$ Francesco Sambiasi, Lingyan lishao (Humilde discusion sobre cuestiones del alma), Biblioteca Apostólica Vaticana, Raccolta Generale Oriente III, 223 (10), Borgia Cinese, 324 (6), Borgia Cinese 364 (12), p. 5. 
vernáculas. Catecismos, breviarios, hagiografías servían a la enseñanza y edificación de los indígenas y apoyaban a los misioneros en su labor apostólica. La redacción de esas obras dependió mucho de la cultura en la cual operaban los misioneros. Fueron numerosas en China donde los padres jesuitas dialogaban con una elite altamente educada y culta. En otras partes donde los indígenas carecían de educación escrita, la publicación de obras servía a la enseñanza de los novicios en los colegios y seminarios y a los misioneros en su labor pastoral. Por otro lado, se usaban también para la formación de los hijos de las elites indígenas laicas en colegios tales como el Colegio franciscano de Santiago Tlatelolco y los Colegios jesuitas de San Gregorio y San Martín de Tepotzotlan para nombrar sólo algunos. ${ }^{39}$ En cuanto a la redacción de catecismos en tierras americanas, los franciscanos fueron los más prolijos con 22 obras, seguidos por los dominicos con 17 y luego los agustinos y jesuitas con 6 obras ambas órdenes. ${ }^{40}$ Los catecismos jesuitas más difundidos fueron los de Gaspar de Astete y Jerónimo de Ripalda (ambos ca. 1591) cuya amplia difusión perduró hasta el siglo Xx. ${ }^{4 \mathrm{I}} \mathrm{El}$ catecismo Limense publicado en Lima en 1595 es otro ejemplo de tales obras. Redactado en castellano por José de Acosta (1539-1600), entre otros autores, fue traducido al quechua y al aymará y ampliamente difundido en Perú, Bolivia, Argentina y Ecuador.

Ahora bien, esta política lingüística de traducción en lenguas vernáculas no se aplicó a los tratados y comentarios aristotélicos y tomistas que se enseñaban en los colegios jesuitas. El acercamiento intelectual europeo a las culturas indígenas que empezó con el inicio de los procesos coloniales y no fue cuestionado hasta el siglo xvin, se basaba en la jerarquía de las culturas según Aristóteles. El orden que clasificaba a las culturas según su progreso desde las bárbaras hasta

\footnotetext{
${ }^{39}$ Robert Ricard, La "conquête spirituelle" du Mexique. Essai sur l'apostolat et les méthodes missionaires des ordres mendiants en Nouvelle-Espagne de 1523-24 à 1572, París, Institut d'ethnologie, 1933, cap. 14.

${ }^{40}$ Nadine Béligand, "Traduire l'évangile et transmettre la foi aux indiens de Nouvelle-Espagne: quelques exemples de catéchismes au service de l'humanisme évangélique”, Revue d'histoire ecclésiastique, 2008, 410.

${ }^{41}$ Sobre la autoría de estas obras véase, Luis Resines, "Astete frente a Ripalda: dos autores para una obra”, Teología y catequesis, núm. 58, 1996, 89-138.
} 
las civilizadas era pensado como natural. Recordemos aquí que, para Aristóteles, el bárbaro es el que no articula, es decir, el que no habla griego. No es de sorprender entonces que los productos intelectuales más elaborados de la cultura europea no se pudieran pensar traducidos en idiomas indígenas. Es la razón por la cual los libros de texto, de Coimbra o la obra de Antonio Rubio, que se enseñaban en los colegios jesuitas se redactaban en latín. Pese a lo anterior, diferente fue la modalidad del encuentro intelectual entre Europa y China en esta misma época. Dos razones pueden explicar este hecho. En primer lugar, los occidentales se representaron a la cultura china como muy desarrollada porque ésta poseía los aspectos que precisamente se valoraban en el Occidente, a saber, una cultura de la escritura y basada en una burocracia estatal centralizada. Esta organización burocrática fundamentada en la escritura es precisamente lo que caracteriza el pensamiento europeo de la modernidad. China, en el momento en que llegaron los jesuitas, presentaba, tanto una extensa cultura escrita, como un desarrollo administrativo, los dos nítidamente superiores a los de la Europa de entonces.

La segunda razón es que los misioneros jesuitas no dominaron los términos de su relación con los letrados chinos. La sociedad que encontraron no necesitaba del "progreso material" ni de la cultura de otra sociedad. Los misioneros tuvieron que ingeniárselas para interesar a los gobernantes chinos y llegar a tener el derecho de practicar su religión en el Imperio del Medio. Fueron varias las estrategias que los jesuitas emplearon en su intento de penetración de la cultura china. La primera iniciativa que tomó Alessandro Valignano (1539-1606), arquitecto de las misiones de Japón y China, fue imponer a los padres el estudio del idioma chino. ${ }^{42}$ Valignano esperaba propiciar una adaptación satisfactoria de los misioneros a la cultura china. Asimismo, los mismos jesuitas que querían introducir su cultura en China tuvieron que empezar por asimilar la cultura de los chinos, quienes imponían un "imperativo cultural cuyo aspecto más importante era

${ }^{42}$ Lo mismo pasó en Japón. Véase, Michael Cooper, s.J., Rodrigues The Interpreter: An Early Jesuit in Japan and China, Nueva York y Tokyo, Weatherhill, 1974, cap. 3. 
la predominancia del chino en los intercambios" ${ }^{43} \mathrm{Y}$ en efecto, durante los siglos XVII y XVIII, eran muy pocos los chinos que estudiaban un idioma extranjero. Por otra parte, Matteo Ricci supo evaluar la importancia que los chinos conferían a los medios escritos y percibió la necesidad de propagar tanto la ciencia como la filosofía europeas por un lado, y conocer y dominar la filosofía china, por otro. Lo anterior constituye la razón por la cual los misioneros jesuitas redactaron tratados aristotélicos en chino.

Una segunda característica que recalca la especificidad del comentario en chino es la adaptación a su pensamiento. Ismael Quiles ha subrayado el hecho de que en la obra de Rubio "la relación con América es poco menos que un accidente. No hay ningún elemento de la filosofía de Rubio que haya sufrido una inflexión o un cambio por virtud de su experiencia americana, salvo el nombre de 'Lógica mexicana'". ${ }^{44}$ Radicalmente opuesto fue el impacto de la cultura china sobre la labor de los misioneros. La adaptación al pensamiento chino tuvo varias vertientes, todas ideadas por Matteo Ricci. En primera instancia, Ricci consideró prudente que fuera progresiva la presentación de la doctrina cristiana a los chinos. Su método catequético se basaba en la distinción entre el catecismo y la doctrina cristiana ${ }^{45}$ Su catecismo, publicado bajo el título Verdadero significado del Señor del Cielo (Tianzhu shiyi, 1603), presentaba los conceptos esenciales del catolicismo tales como la existencia de Dios y la retribución del bien y del mal mientras que la Doctrina del Señor del Cielo (1605) explicaba en su totalidad la doctrina cristiana y era exclusivamente reservada a los catecúmenos y creyentes.

Esta estrategia también operaba una distinción entre la revelación natural que se podía entender mediante la razón y las verdades sobrenaturales a las cuales solamente se podía acceder por acto de fe.

${ }^{43}$ Erik Zürcher, "Jesuit Accommodation and the Chinese Cultural Imperative" en David E. Mungello, The Chinese Rites Controversy: Its History and Meaning, Nettetal, Steyler, 1994, 41.

${ }^{44}$ Citado por Torchia Estrada, "El Padre Antonio Rubio y la enseñanza filosófica..." p. 30; Ismael Quiles, "Ubicación de la filosofía del P. Antonio Rubio, s.I., dentro de la historia de las escolásticas" Ciencia y fe, vol. 27, núm. 1, 1951.

${ }^{45}$ Para este párrafo, véase Gianni Criveller, Matteo Ricci, Missione e ragione: una biografia intelletuale, Milán, Pimedit, 2010, 68-74. 
Era una distinción tradicional entre teología natural y teología revelada que se enseñaba en todos los seminarios de la Compañía de Jesús. La obra de Sambiasi no ignora esta distinción en la medida en que el orden de exposición sigue la teología natural, en particular, la Cuarta Vía de exposición tal como Tomás de Aquino la definió en la Suma teológica:

La cuarta [vía] se deduce de la jerarquía de valores que encontramos en las cosas. Pues nos encontramos que la bondad, la veracidad, la nobleza y otros valores se dan en las cosas. En unas más y en otras menos. Pero este más y este menos se dice de las cosas en cuanto que se aproximan más o menos a lo máximo. Así, caliente se dice de aquello que se aproxima más al máximo calor. Hay algo, por tanto, que es muy veraz, muy bueno, muy noble; y, en consecuencia, es el máximo ser; pues las cosas que son sumamente verdaderas, son seres máximos, como se dice en II Metaphys. Como quiera que en cualquier género, lo máximo se convierte en causa de lo que pertenece a tal género -así el fuego, que es el máximo calor, es causa de todos los calores, como se explica en el mismo libro-, del mismo modo hay algo que en todos los seres es causa de su existir, de su bondad, de cualquier otra perfección. Le llamamos Dios. ${ }^{46}$

Si analizamos la obra de Sambiasi, vemos que empieza su descripción del alma por la parte epistemológica para gradualmente llegar a las implicaciones teológicas de la noción en las cuales reside su verdadera meta. Este modo de exposición empezaba por los argumentos más familiares para los chinos y así el autor podía paulatinamente introducirlos a doctrinas más específicas del cristianismo y que les eran a la vez más extrañas. En este sentido, la descripción de las potencias y funciones del alma hacía eco de teorías propias de los pensadores chinos sobre la mente (xin), las afecciones (qing) o las potencias (nengli) entre otras. Al otro extremo de la exposición, la noción cristiana de Dios era absolutamente extrańa al pensamiento chino, y es por esta razón que el Lingyan lishao termina con la exposición de la semejanza del alma con Dios.

${ }^{46}$ Suma Teológica part, 1, q. 2, art. 3, obj. 2. 
Por otra parte, el método de acomodación de Matteo Ricci lo llevó a presentar al cristianismo como una doctrina cercana al confucianismo y a asociarlo con la enseñanza científica. La estrategia empezó como una alianza con los confucianos en contra de los budistas y con la idea de que la cultura china carecía de una verdadera religión. ${ }^{47}$ Con el tiempo, Ricci llegó a atribuirle al confucianismo valores éticos y sociales. Terminó por acertar que los chinos habían conocido la noción de Dios, la cual estaba presente en las obras más tempranas del confucianismo bajo el nombre de Shangdi (Soberano en lo alto) o Tian (Cielo). ${ }^{48}$ Asimismo, en el Verdadero significado del Señor del Cielo, el letrado occidental dice "aunque llegué a la China tarde en mi vida, he estudiado con aplicación las obras antiguas de China y he descubierto que los hombres superiores de los tiempos antiguos adoraban y reverenciaban al Soberano en lo Alto, [el Supremo Señor] del Cielo y de la tierra". En la misma vena, dice: "El que llaman el Señor del Cielo en mi humilde país es El que llaman Shangdi (Soberano en lo Alto) en chino". ${ }^{49}$ Ricci también comprobaba su argumento citando a los clásicos confucianos como el Libro de las odas, el Yijing, el Libro de los ritos y la Doctrina del medio entre otros. Lo que interesaba a Matteo Ricci era la nítida referencia a un dios antropomórfico presente en estas obras. Leemos por ejemplo: "Nuestro Señor del Cielo es el Soberano en lo Alto que se menciona en los antiguos escritos canónicos [chinos] [como lo comprueba el texto siguiente]: Citando a Confucio, la Doctrina del Medio dice que 'Las ceremonias de los sacrificios al Cielo y la Tierra son para el servicio del Soberano en lo Alto", ${ }^{50}$ Para Ricci era "bastante claro que el Soberano en lo Alto y el Señor del Cielo solamente diferían en nombre". ${ }^{51}$

Otra consecuencia del énfasis en el confucianismo fue la adopción de un vocabulario confuciano en la redacción de los tratados

${ }^{47}$ Paul A. Rule, K'ung-tzu or Confucius? The Jesuit interpretation of Confucianism, Sydney, Londres, Boston, Allen \& Unwin, 1986, 26-34.

${ }^{48}$ Matteo Ricci, Tianzhu shiyi, traducción y notas Douglas Lancashire y Peter $\mathrm{Hu}$ Kuo-chen, St Louis, Institute of Jesuit Sources, 1986, chap. II, en particular $121 \mathrm{ff}$.

${ }^{49}$ Tianzhu shiyi, $\$ 78,107$ y $\$ 103,121$.

${ }^{50}$ Tianzhu shiyi, $\$ 104,122-23$.

${ }^{51}$ Tianzhu shiyi, $\$ 108,124-25$. 
jesuíticos en chino. Esta decisión se debió, en gran medida, al hecho de que Ricci no quería usar palabras transliteradas que tuvieran una consonancia bárbara (regresamos a las sonoridades no articuladas de Aristóteles pero trasladadas al chino) lo que ofendería el gusto literario de los letrados. Esta decisión tuvo consecuencias graves para el devenir de la misión de China y de la Compañía de Jesús en general. Pocos ańos después de la muerte de Matteo Ricci, se alzó el problema de la inadecuación de los términos chinos para referirse a la sustancias espirituales del cristianismo. Niccoló Longobardo (1559-1654), en particular, subrayó que un cierto materialismo era inherente a toda la filosofía china por lo cual las palabras usadas para traducir el nombre de Dios, el alma y el espíritu tenían connotaciones materiales evidentemente contrarias a su significado teológico. Longobardo dedicó un tratado completo al problema, el Resposta breve sobre as Controversias do Xámty, Tiēnxîn, Lîmhoên, e outros nomes e termos sinicos: per se determinar quaes delles podem ou nao podem usarse nesta Xrandade. Dirigida aos Padres das Residencias da China, pera a verem, a depois emviare com ou seu parecer sobre ella ao Nosso Padre Visitador (1623-1624?). Este hecho desató la Controversia de los Términos y después de 1630 la Controversia de los Ritos.

Por último, los jesuitas tendieron a favorecer las obras que más podían interesar a los chinos. En este sentido se hizo particular énfasis en la filosofía moral. El Tratado de la amistad de Matteo Ricci (Jiaoyou lun, 1595) es un buen ejemplo de una obra cuyo tópico era de interés tanto para los occidentales como para los letrados chinos. Para esta obra, Matteo Ricci se apoyó libremente en su cultura humanista sabiendo que llamaría la atención de los confucianos chinos porque la amistad constituía una de las cinco relaciones primordiales del confucianismo. ${ }^{52}$ Lo mismo se puede decir de un libro suyo cuyo tema es totalmente diferente: el Método mnemónico del occidente (Xiguo jifa, 1596) porque, de igual manera, presentaba características propias de la educación china esencialmente basada en la memorización de textos. Sambiasi también trató este último tema

\footnotetext{
${ }^{52}$ Las cinco relaciones confucianas son gobernante-subdito; padre-hijo; esposomujer; hermano mayor-hermano menor y amigos.
} 
en su tratado del alma. ${ }^{53} \mathrm{El}$ Estudio occidental de la realización de sí (Xiushen xixue, 1636) de Alfonso Vagnoni es una obra con un contenido filosófico un poco más árido y preciso, pero que no carecía de interés para los letrados chinos en la medida en que trataba de la ética, género central de la filosofía china. El mismo tratado de Sambiasi podía llamar la atención de un lector chino educado ya que el tema del alma pertenece de manera difusa a la literatura china y al pensamiento budista. Todas las características mencionadas refieren precisamente a lo que se llama la acomodación mediante la cual los padres misioneros intentaron llevar su mensaje evangélico a China.

\section{Conclusión}

Alison Simmons ha subrayado el hecho de que no hay una sola lectura jesuítica de Aristóteles, sino más bien un estilo de los comentarios jesuíticos que se traduce por una presentación extremadamente clara y ordenada del pensamiento del Estagirita. ${ }^{54}$ Sin embargo, las diferencias en las interpretaciones de la doctrina del alma propuestas por Antonio Rubio, Manuel de Goís y Francesco Sambiasi, en particular, la diferencia en la importancia que cada uno atribuye al alma vegetativa y a la voluntad, son ilustrativas de la cierta libertad que gozaban los padres jesuitas en su interpretación de la filosofía tanto aristotélica como tomista. ${ }^{55}$ También esto demuestra que no se puede hablar de una filosofía jesuítica en general.

Finalizaremos esta conclusión con unas palabras sobre el uso de las lenguas vernáculas en tierras misioneras. Danièle Dehouve ha mostrado en su obra sobre el uso de los exempla en la evangelización de México, cómo los misioneros, jesuitas en particular, tuvieron, en un primer momento, que apropiarse la retórica y semántica náhuatl. Sin embargo, es notable el hecho de que las estructuras de las sentencias en sus traducciones al náhuatl sigan un patrón calcado del latín negando así las características del pensamiento indígena.

${ }^{53}$ Lingyan lishao, pp. 28-42 y en particular, p. 41.

${ }^{54}$ Simmons, "Jesuit Aristotelian Education..." p. 526.

${ }^{55}$ Ibid., p. 534. 
Operaron también un trabajo de "reelaboración semántica" de ciertas palabras. El déni de différence (negación de la diferencia), del cual nos habla Danièle Dehouve, hace que

a partir del siglo XviI los eclesiásticos pueden hacer abstracción de lo extraño en los usos indígenas y descifrar tranquilamente sus palabras y sus actos de acuerdo con los referentes proveídos por el dogma católico. [...] Estos esfuerzos de los jesuitas de México y del mundo entero en los siglos xvir y XVIII son llamativos por su etnocentrismo. Todos los hechos de lengua-retórica, semántica, narraciones-son percibidos a través de referencias aprendidas en los seminarios. Cada palabra y cada idea son cuidadosamente aisladas del contexto cultural que podría darles sentido. ${ }^{56}$

Por el contrario, hemos visto que en su trabajo exegético y pedagógico, los misioneros en China debieron asimilar e integrar a su quehacer literario e intelectual elementos de la cultura china, empezando con el idioma y la filosofía. En este punto reside la característica más distintiva de los comentarios redactados en chino.

\section{BiBLIOGRAFÍA}

Andrade, Antonio Alberto de, Curso Conimbricense. P. Manuel de Gois, Moral a Nicómaco, de Aristóteles, Introducción, Lisboa, Instituto de Alta Cultura, 1957, XVI-XIX.

BÉLIGAND, Nadine, “Traduire l'évangile et transmettre la foi aux indiens de Nouvelle-Espagne: quelques exemples de catéchismes au service de l'humanisme évangélique", Revue d'histoire ecclésiastique, 2008, 410.

Carvalho, Mario Santiago de, "Tentâmen de sondagem sobre a presença dos platonismos no volume do De Anima do primeiro Curso Jesuíta Conimbricense" en José Antônio de Camargo Rodrigues de Souza, Idade Média: tempo do mundo, tempo do homens, tempo de Deus, Porto Alegre, Est Ediçóes, 2006, 391.

${ }^{56}$ Danièle Dehouve, L'évangélisation des Aztèques ou le pécheur universel, París, Maisonneuve \& Larose, 2004, 271. 
, Psicologia e ética no curso jesuita conimbricense, Lisboa, Edições Colibri, Forum de Ideias, 2010, 31.

Commentarii Collegii Conimbricensis S. I. in duos libros de generatione et corruptione Aristotelis Stagiritae, Coimbra, A. Mariz, 1597.

Commentarii Collegii Conimbricensis S. I. in libros Aristotelis qui Parva Naturalia appellantur, Lisboa, S. Lopes, 1593.

Commentarii Collegii Conimbricensis S. I. in libros Metereororum Aristotelis Stagiritae, Lisboa, S. Lopes, 1593.

Commentarii Collegii Conimbricensis S. I. in tres libros De anima Aristotelis Stagiritae, Coimbra, A. Mariz, 1598.

Commentarii Collegii Conimbricensis S. I. in universam Dialecticam Aristotelis, Coimbra, D. G. Loureiro, 1606.

Commentarii Collegii Conimbricensis Societatis Iesu in quattuor libros De Coelo Aristotelis Stagiritae, Lisboa, S. Lopes, 1593.

Commentarii Collegii Conimbricensis Societatis Jesu in octo libros Physicorum Aristotelis Stagyritae, Coimbra, A. Mariz, 1592; Lyon, 1594.

Commentarii Colegii Conimbricensis, Societatis Iesu: In tres libros de Anima, Aristotelis Stagiritae, libro I, cap.1: "Scientiae de anima dignitas: methodus inueniendi animae definitionem: ac modus, quem in definiendo seruent Physiologus, Medicus, Dialecticus, et Matematicus".

Cooper, Michael s. J., Rodrigues The Interpreter: An Early Jesuit in Japan and China, Nueva York y Tokyo, Weatherhill, 1974, cap. 3.

Criveller, Gianni, Matteo Ricci, Missione e ragione: una biografia intelletuale, Milán, Pimedit, 2010, 68-74.

DucEux, Isabelle, La introducción del aristotelismo en China a través del De anima, siglos XVI-XVII, México, El Colegio de México, 2009, 63-66.

Dehouve, Danièle, Lévangélisation des Aztèques ou le pécheur universel, París, Maisonneuve \& Larose, 2004, 271.

Falcon Gyves, Camilo, El P. Antonio Rubio, S. J. (1548-1615): sus comentarios a los libros "De anima" de Aristóteles, México, Bajo el signo de "Ábside", 1945, 22.

Gilson, Etienne, La filosofía en la Edad Media, Madrid, Gredos, 1985, 341-342. 
Grendler, Paul F., "The Universities of the Renaissance and Reformation" Renaissance Quarterly, vol. 57, núm. 1, 2004, 25-26.

Holtz, Louis, "Le rôle des commentaires d'auteurs classiques dans l'émergence d'une mise en page associant texte et commentaire (Moyen Âge occidental)" en Marie-Odile Goulet-Cazé, Tiziano Dorandi, coords., Le commentaire entre tradition et innovation, Actes du Colloque International de l'Institut des Traditions Textuelles, París, Villejuif, 22-25 octubre 1999, París, Librairie Philosophique J. Vrin, 2000, 101-116.

In libros Ethicorum Aristotelis ad Nicomachum aliquot Conimbricensis Cursus disputationes, in quibus praecipua quaedam Ethicae disciplinae capita continentur, Lisboa, S. Lopes, 1593.

Koyré, Alexandre "Aristotélisme et platonisme dans la philosophie du moyen âge”, Études d'histoire de la pensée scientifique, París, Gallimard, Tell, 1973, 39.

Libera, Alain de, "De la lecture à la paraphrase; remarques sur la citation au Moyen Âge”, Langage, núm. 73, 1984, 17-29.

LoHr, Charles, "Les jésuites et l'aristotélisme du Xvie siècle" en Luce Giard, Les jésuites à la Renaissance: système éducatif et production du savoir, París, PUF, 1995, 80.

Martins, Antonio M., "The Conimbricenses. Introductory note to the Commentarii Collegii Conimbricensis Societatis Iesu" Biblioteca Saavedra Fajardo de Pensamiento Político Hispánico, p. 2.

PARK, Katharine, "The organic Soul” en Charles B. Schmitt, Quentin Skinner y Eckart Kessler, The Cambridge History of Renaissance Philosophy, Cambridge, Cambridge University Press, 1998, 473.

PARK, Katharine y Eckard Kessler, "The Concept of Psychology” en The Cambridge History of Renaissance Philosophy, Cambridge, Cambridge University Press, 1998, cap. 13.

Quiles, Ismael, "Ubicación de la filosofía del P. Antonio Rubio, S.I., dentro de la historia de las escolásticas" Ciencia y fe, vol. 27, núm. 1, 1951.

Redmond, Walter, "Self-Awareness in Colonial Latin American Philosophy”, Jahrbuch für Geschichte Lateinamerikas 41, Colonia, Weimar, Viena, Böhlau Verlag, 2004, 370. 
Resines, Luis, “Astete frente a Ripalda: dos autores para una obra”, Teología y catequesis, núm. 58, 1996, 89-138.

Ricard, Robert, La "conquête spirituelle" du Mexique. Essai sur l'apostolat et les méthodes missionaires des ordres mendiants en Nouvelle-Espagne de 1523-24 à 1572, París, Institut d'ethnologie, 1933, cap. 14.

Ricci, Matteo, Tianzhu shiyi, traducción y notas Douglas Lancashire y Peter Hu Kuo-chen, St Louis, Institute of Jesuit Sources, 1986, cap. II, en particular $121 \mathrm{ff}$.

Rubio, Antonio, Lógica, "Auctoris ad lectorem praefatio".

Rule, Paul A., K'ung-tzu or Confucius? The Jesuit interpretation of Confucianism, Sydney, Londres, Boston, Allen \& Unwin, 1986, 26-34.

SAmbiasi, Francesco, Lingyan lishao (Humilde discusion sobre cuestiones del alma), Biblioteca Apostólica Vaticana, Raccolta Generale Oriente III, 223 (10), Borgia Cinese, 324 (6), Borgia Cinese 364 (12), p. 5.

Schmitт, Charles, Aristotle and Renaissance, Cambridge, Harvard University Press, 1983, 92.

Simmons, Alison, "Jesuit Aristotelian Education: The De anima Commentaries" en John W. O'Malley, Gauvin Alexander Bailey, Steven J. Harris y T. Frank Kennedy, eds., The Jesuits: Cultures, Sciences, and the Arts, 1540-1773, Toronto, Bufalo, Londres, University of Toronto Press, 1999, 526.

Torchia Estrada, Juan Carlos, "El Padre Antonio Rubio y la enseñanza filosófica de los jesuitas en la Nueva Espańa”, CUYO, Anuario de Filosofía Argentina y Americana, núm. 13, 1996, 22-23.

Zürcher, Erik, "Jesuit Accommodation and the Chinese Cultural Imperative" en David E. Mungello, The Chinese Rites Controversy: Its History and Meaning, Nettetal, Steyler, 1994, 41.

FECHA DE RECEPCIÓN DEL ARTíCUlO: 11 de marzo de 2011

FECHA DE ACEPTACIÓN Y RECEPCIÓN DE LA VERSIÓN FINAL: 5 de OCtubre de 2011 